\title{
Intrauterine Cordocentesis
}

National Cancer Institute

\section{Source}

National Cancer Institute. Intrauterine Cordocentesis. NCI Thesaurus. Code C92820.

The retrieval of blood sample from the umbilical cord while the fetus is in utero. 